\title{
Corona und die Alten - um wen sorgen wir uns wirklich?
}

\author{
Stefan Dinges
}

\section{Einleitung}

Die chronisch kritischen Rahmenbedingungen und strukturellen Ungerechtigkeiten in der stationären Altenhilfe treten unter den krisenhaften Bedingungen einer Pandemie deutlich zutage. Die Herausgeber dieses Bandes haben sich eine essayistische Kommentierung der Corona-Krise in Bezug auf ihre Auswirkungen in der stationären Altenhilfe gewünscht, und zwar vor dem Hintergrund des pflegeethischen Diskussionspapiers der Akademie für Ethik in der Medizin vom 12. Mai 2020․․ Die Form des Essays erlaubt es, einige Thesen zur Diskussion zu stellen und als Grundlage eher auf aktuelle Medienberichte und Stellungnahmen zurückzugreifen denn auf Studien und Literatur. Die skizzenhaften Überlegungen zielen auf vertiefende Reflexionen und weiterführende Themen sowie Begründungen für Lösungsansätze. Dazu hier einige Eingangshypothesen:

- Die Erfahrungen in der stationären Altenhilfe, sowohl mit den Herausforderungen der Pandemiekrise wie mit ihren chronisch fragilen Rahmenbedingungen, brauchen dringend mehr pflegewissenschaftliche und pflegeethische Reflexion und Forschung. Das Medizinsystem hat mit einer Flut von Forschungspublikationen auf das neue Virus reagiert. Jene Auswirkungen, die z. B. Isolation sowie fehlende Berührungen und Besuche auf alte Menschen haben, sind von Seiten der Pflegewissenschaft bereits gut erforscht - und trotzdem fanden diese Studienergebnisse kaum Berücksichtigung in den Entscheidungen und Empfehlungen für die (stationäre) Altenhilfe.

- Die Stärken und Schwächen im Umgang mit der Krise sind bedingt durch eine chronifizierte strukturelle Ungerechtigkeit in der Verteilung der Ressourcen (z. B. Finanzen, Personal, Zeitbudgets, Aufmerksamkeit, gesellschaftliche Mitverantwortung) in den deutschsprachigen Ge-

1 https://www.aem-online.de/fileadmin/user_upload/2020_05_12_Pflegeethische_Re flexion_Papier.pdf [21.06.2020]. 
sundheitssystemen. Eine Veränderung hierin wird seit mehr als zehn Jahren gefordert und versprochen - und nicht eingelöst. ${ }^{2}$

- Aus diesem Misstrauensvorschuss gegenüber der Gesundheitspolitik heraus ist zu überprüfen, ob die vorgegebenen Werte ,Schutz und Gesundheit der Risikogruppen' und ,Schutz und Sicherheit des Gesundheitssystems ' wirklich handlungsleitend sind und waren - und welche Wirkung und Erfolg sie erreicht haben.

- Die Corona-Krise ist der Auslöser, nicht die Ursache für die Erfahrungen in der Pflege und in den Altenpflegeeinrichtungen des Frühjahrs 2020 - und verdeutlicht einen dringenden Veränderungsbedarf in den Einrichtungen und im Gesundheitssystem. Gleichzeitig wurde sichtbar, wie schnell kulturelle Errungenschaften in den Gesundheitseinrichtungen (z. B. geteilte Entscheidungsfindung, Ethikberatung, Vorsorgedialog, Palliative Kultur, Sterbebegleitung etc.) in der Krise zur Disposition stehen bzw. nicht durchgehalten werden können.

- Aus der positiven Krisenbewältigung (z. B. mobile Testungen zuhause, Vermeiden von unnötigen Krankenhauseinweisungen, vorausschauende Therapiezielplanungen mit den Patient*innen) lassen sich Konzepte und Bausteine für eine bessere Versorgung alter und hochaltriger Menschen zuhause und in den Pflegeeinrichtungen ableiten: Neben der Notarztversorgung bräuchte es mobile geriatrisch-palliative Dienste (Medizin/Pflege), die ad hoc vor Ort alte Menschen so versorgen können, dass sie zuhause oder im Heim bleiben können.

- Die Gesundheitsberufe, insbesondere die Pflegenden, haben motiviert einen hervorragenden Job in der Krise gemacht - und haben es nicht verdient, dass ihnen die moralische Verantwortung für die ökonomischen Knappheiten und andere Rahmenbedingungen, Konflikte und Fehler angelastet werden, für die im weitesten Sinne die Gesundheitspolitik verantwortlich ist. Doch die Mitarbeiter*innen im Gesundheitswesen nehmen es persönlich und reagieren mit moralischem Stress. ${ }^{3}$ Das gilt für die Pflegenden ebenso wie für die Mediziner*innen und alle anderen Gesundheitsberufe. Gerade der Umgang mit alten Menschen in der Pandemiekrise wirft Fragen und Wertkonflikte auf, die vordringlich im Gesundheitssystem, in den Organisationen und in der Gesellschaft zu bearbeiten sind. Das Modell der individuellen Verant-

2 Zuletzt suchte in Österreich die parlamentarische Enquete „Würde am Ende des Lebens“ 2014/15 entsprechende Ansätze: https://www.parlament.gv.at/PAKT/VHG /XXV/I/I_00491/fname_386917.pdf [21.06.2020].

3 Vgl. Dinges, Ethikberatung als Prävention; Dinges, Eine Sorge für das Ganze. 
wortung ${ }^{4}$ (das von Pflegenden und Ärzt*innen oft thematisiert wird) kommt bei globalen Herausforderungen an seine Grenzen und braucht eine kollektive Ergänzung, um eine Überforderung der Einzelnen zu vermeiden.

\section{Einblicke in die Praxis der Gesundheitseinrichtungen}

Zu Beginn des Pandemieausbruchs Anfang März 2020, nach der rasanten, eskalierenden Entwicklung der Covid-19-Erkrankungen vor allem im Norden Italiens, lag die Aufmerksamkeit der Öffentlichkeit und im Gesundheitswesen insbesondere auf den Krankenhäusern und den Beatmungsressourcen der Intensivstationen: Wie und wo sollen die Infizierten ausreichend versorgt werden? Im Fokus waren die Risikogruppen, Menschen in hohem Alter und mit Vorerkrankungen, die es zu schützen und bei Infektion bestmöglich zu versorgen galt. Zu diesem Zeitpunkt war noch nicht klar, wie sich die Altersverteilung der Infizierten und wie sich der Krankheitsverlauf gestalten würde. Die getroffenen Maßnahmen sind bekannt: Der operative Betrieb mit elektiven Eingriffen wurde heruntergefahren, nur noch Notfälle behandelt, sogar OP-Räume wurden als mögliche Beatmungsplätze vorbereitet und die Eingänge in die Krankenhäuser mit strikten Zugangskontrollen versehen. In Wien beispielsweise wurden Spitäler in Covid/Non-Covid aufgeteilt, um Infektionsketten zu vermeiden: Zu bedrohlich und zu traumatisch waren die Fernsehbilder und die Erfahrungsberichte aus Norditalien. Gleichzeitig wurde in den Krankenhäusern und auf Trägerebene darüber nachgedacht, was mit Patient*innen geschehen sollte, die einen benötigten Beatmungsplatz nicht bekommen konnten. Es stellte sich die Frage nach Entscheidungs- und Zuteilungskriterien für eventuell zu knappe Ressourcen. Der Hinweis von medizinischen Fachgesellschaften ${ }^{5}$, dann jedenfalls für angemessene Palliativtherapie und Sterbebegleitung zu sorgen, erschien vorschnell und beschrieb gleichzeitig den drohenden Verlauf der Infektion, besonders in den Risikogruppen. Auf in-

4 Als Beispiel die Einigkeit von Medizin und Recht, auf medizinische Einzelentscheidungen und -verantwortung zu fokussieren: https:/www.addendum.org/coronavir us/triage-oesterreich/ [21.06.2020].

5 Für Österreich ÖGARI: https://www.anaesthesie.news/wp-content/uploads/OEGA RI-ICU-Therapy-Guideline-1.pdf [21.06.2020] und für Deutschland DIVI: https:/w ww.divi.de/empfehlungen/publikationen/covid-19/1549-entscheidungen-ueber-diezuteilung-intensivmedizinischer-ressourcen-im-kontext-der-covid-19-pandemie-klin isch-ethische-empfehlungen/file [21.06.2020]. 
ternen Kliniken wurde z. B. darüber nachgedacht, überbrückend eine Maskenbeatmung anzubieten, falls ein benötigtes Intensivbett nicht verfügbar wäre. Gleichzeitig wurde in den einzelnen Einrichtungen und auf Trägerebene darum gerungen, wie einerseits Rationierungs- und TriageEntscheidungen verantwortlich getroffen (durch Intensivkoordinator*innen auf Haus- und Trägerebene) und andererseits wie solche Entscheidungsprozesse fachlich und ethisch transparent und begründet gestaltet werden können (in Form eines Mehr-Augen-Prinzips, interprofessionell, mit Unterstützung von ethischen Beratungsstrukturen).

In den ersten beiden Märzwochen kamen aus Italien und Frankreich neue schlechte Nachrichten:

- Die Sterbezahlen mussten in Norditalien um ca. 30 bis 40 Prozent nach oben korrigiert werden, weil die Sterbefälle in den Pflegeheimen noch nicht aufgenommen waren ${ }^{6}$;

- In Frankreich wurde darüber diskutiert, pauschal Pflegeheimbewohner*innen über 80 Jahren nicht mehr für eine intensive Beatmungstherapie vorzusehen ${ }^{7}$ (auch wenn das nicht als valide Information bestätigt werden konnte);

- In Deutschland ${ }^{8}$ und Österreich wurde berichtet, dass mehr als 30 Prozent der Infektionen in den Einrichtungen der stationären Altenhilfe stattgefunden hatten und dort auch bis zu 30 Prozent der Sterbefälle zu verzeichnen waren - durch fehlende Tests bislang nur eine Schätzung, da nur vorher positiv getestete Personen als Covid-19-Sterbefall gezählt wurden.

Anfang April 2020 war die Welle von mit Covid-19 infizierten Patient*innen noch nicht eingetroffen und wurde bis Ostern erwartet, um dann gänzlich auszubleiben: Nur 15 bis 25 Prozent der vorbereiteten Ressourcen im Intensivbereich wurden benötigt; auf den Allgemeinstationen von Privatspitälern wurde zeitweise Kurzarbeit ${ }^{9}$ eingeführt, in den Allgemeinspitälern Resturlaub abgebaut. Schon im Rahmen der Triage-Planungen und expliziten Rationierungs- bzw. Priorisierungsmaßnahmen waren die Bewohner*innen der stationären Langzeitpflege besonders in den Blick gera-

6 https://www.n-tv.de/panorama/Wurden-Italiens-Senioren-im-Stich-gelassen-article2 1700380.html [21.06.2020].

7 https://www.faz.net/aktuell/politik/inland/im-elsass-werden-alte-corona-patienten-n icht-mehr-beatmet-16698139.html [21.06.2020].

8 https://www.sueddeutsche.de/politik/coronavirus-altenheim-tote-1.4884742 [21.06.2020].

9 Nur 30 Prozent Auslastung: https://tirol.orf.at/stories/3042830/ [21.06.2020]. 
ten. Ihnen galt die größte Sorge und gleichzeitig wurden sie im Falle einer Pandemiewelle als ein großes Risiko für die Spitäler und für die Gesundheitsversorgung gesehen: Mit Patientenverfügungen, Therapiezielprotokollen und Scores sollte die Aufnahme hochaltriger, multimorbider Menschen bei einem Covid-19-Verdacht in die Krankenhäuser vermieden und diese nach Möglichkeit in den eigenen Einrichtungen versorgt werden.

Dass alte und hochaltrige, multimorbide Menschen zur Hochrisikogruppe mit hohem Infektionsrisiko gehören, hat vielerorts zu strengen, ja rigiden Reaktionen geführt: Einrichtungen der stationären Langzeitpflege verhängten auf behördliche Anordnung oder aus eigenem Antrieb konsequente Besuchs- und Betretungsverbote, sei es für Angehörige oder auch z. B. für mobile Dienste in der Palliativversorgung oder der Physiotherapie etc., die nicht mehr in die Einrichtungen gelassen wurden. In vielen Pflegeeinrichtungen wurden nicht nur alle Besuche unterbunden, sondern auch für die Bewohner*innen de facto eine Ausgangssperre verhängt. Sie durften ihre Zimmer und die Einrichtung nicht verlassen. Den Angehörigen wurde trotz Zusicherung von Sicherheitsvorkehrungen und Schutzmaßnahmen verweigert, die pflegebedürftigen Menschen zu einem kurzen Spaziergang auf dem Gelände an der Tür abzuholen. ${ }^{10}$ Wenn Bewohner*innen trotzdem z. B. für einen Arztbesuch die Einrichtung verließen, wurden sie in ihrem Zimmer für 14 Tage unter Quarantäne gestellt.

Durch die Besuchsverbote wurde - nicht nur in End-of-Life-Situationen und in Sterbebegleitungen - eine adäquate Versorgung und Begleitung durch Verwandte und Freunde, aber auch durch mobile Dienste und Ehrenamtliche unterbunden. Für viele Pflegende und Leitungsverantwortliche stellte das eine Herausforderung, Zerreißprobe und oft auch moralische Belastung dar. Die Unterstützung seitens der Angehörigen und durch Ehrenamtliche in der psychosozialen Begleitung, im Rahmen der Mobilisation durch Spaziergänge, aber auch als Unterstützung bei den Mahlzeiten, war gänzlich weggebrochen. Insbesondere die psychosoziale Begleitung, aber auch der alltägliche Spaziergang konnte von den Pflegenden nur unvollständig kompensiert werden. Die sichtbare Reduktion der Mobilität führte in kürzester Zeit zu körperlichen Einschränkungen, Mobilitätsverlusten, aber auch zu einer Reduktion kognitiver Beweglichkeit. Für die Pflegenden ist die körperliche und geistige Reduktion als Folge der Isolation, insbesondere angesichts ihres professionellen Auftrags, nur schwer

10 Vgl. das Positionspapier der Bundesinteressenvertretung für alte und pflegebetroffene Menschen e.V. (BIVA-Pflegeschutzbund) vom 26.03.2020 unter https://www. biva.de/positionspapier-besuchsbeschraenkungen-pflegeheim/ [21.06.2020]. 
auszuhalten - sie fühlen sich für die Verschlechterungen des Allgemeinzustandes verantwortlich, sie fühlen sich (mit)schuldig.

\section{Reflexionsorte in der Covid-19-Krise}

Wo ergaben sich für Medizin- und Organisationsethik Möglichkeiten des Anknüpfens und der Bearbeitung in der Krise? Ein Leitsatz der Krisenkommunikation behauptet ja, in der Krise trägt nur das, was zuvor etabliert und geübt wurde. Hier einige kurze persönliche Einblicke aus der klinischen Praxis:

In einem Krankenhaus eines konfessionellen Trägers wurden die vorhandenen lokalen Ethikstrukturen, ein Klinisches Ethikkomitee (KEK) und lokale Ethikberatung weitgehend ausgesetzt. Die Krisenentscheidungen wurden von der Führung des Hauses bzw. von der Holding getroffen. Prioritär waren die fachlichen Kompetenzen aus Medizin und Pflege gefragt, mit den Infektionen umzugehen bzw. die anderen Gesundheitsberufe dort einzubinden, wo es Behandlung und Versorgung im Alltag notwendig machten: bei Palliativpatient"innen und auf der Geburtsstation. Wichtig war auch, in die Wien-weite Koordination der Intensivressourcen eingebunden zu werden. Die Treffen des KEKs dagegen wurden für ein Vierteljahr ausgesetzt. Eine Vermutung: Die ethischen Herausforderungen und Fragen wurden so gut wie möglich von den Mitarbeiter"innen übernommen und in den erlernten Routinen persönlich bearbeitet; Unterstützung von außen oder durch Ethikkonsile wurde nicht angefragt.

Ganz anders bei einem großen kommunalen Träger, der seit einem halben Jahr in einem Pilotprojekt mit der formalen Etablierung von Klinischer Ethikberatung in einem Krankenhaus begonnen hatte: Hier wurde ein akuter, direkter ethischer Reflexionsprozess in der Weichenstellung für die Krisenbearbeitung angefragt, sowohl auf Ebene lokaler Krisenstäbe im Umgang mit Triage- und Versorgungsentscheidungen wie auch auf Trägerebene in der Beratung effizienter Entscheidungsstrukturen zur Krisenbewältigung. Auf beiden Ebenen wurde ein Bedarf an ethischer Unterstützung ausgemacht: Auf Einrichtungsebene wurde in der klinischen Ethikberatung ein Instrument gesehen, konflikthafte Versorgungsentscheidungen zu begleiten; auf Träger- und Einrichtungsebene wurden interprofessionelle Ethikboards (teilweise aus vorhandenen informellen Ethikzirkeln und Gruppen gebildet) nachgefragt und ad hoc einberufen, mitunter mit noch zu klärenden Aufgaben und Befugnissen.

Inwieweit Strukturen und Ressourcen von Ethikberatung in den Einrichtungen und Diensten der Altenpflege zum Einsatz kamen - so sie 
überhaupt existent sind -, ist nicht bekannt. Die Vermutung ist, auch hier mussten die Pflegenden mit ihren Führungsverantwortlichen auf der Mikroebene entscheiden bzw. mit den internen und externen Vorgaben zurechtkommen. Ein positives Beispiel stellt z. B. die Hilfe im Alter dar, ein Altenhilfeträger der Inneren Mission München. Der trägerweite Ethikbeirat hat gerade eine Stellungnahme zur Coronakrise ${ }^{11}$ veröffentlicht, die neben guten Gründen für das Vorgehen in den Einrichtungen auch die Forderung nach gesundheitspolitischer Unterstützung veröffentlichte.

Parallel begannen sich die deutschsprachigen Medizinethiker"innen und Ethikberater*innen in wöchentlichen Videokonferenzen zu vernetzen. Der erste Themenschwerpunkt war eine mögliche Triage/Zuteilung der Intensivressourcen im Falle von Knappheit. Schon beim dritten Treffen wurde, da die Krise nicht zu den Intensivstationen gelangte, die Situation in den Altenpflegeheimen zum Thema - entzündet an den allgemeinen Besuchsregelungen in den Krankenhäusern und Heimen. Viele der Beteiligten suchten schnell und lösungsorientiert nach ,models of good practice', um mit den Vorgaben und Auflagen umzugehen. Vielfach wurden Mut und Flexibilität eingefordert - ganz im aristotelischen Modell der Tugendethik -, um die Krise, die Konflikte und Wertverstöße individuell zu bearbeiten.

\section{Diskussionspapier und pflegeethische Reflexionen der AEM}

Die beiden Arbeitsgruppen „Pflege und Ethik“ der Fachgesellschaft Akademie für Ethik in der Medizin (AEM) hatten sich angesichts dieses Diskussionsverlaufs entschlossen, dieser pragmatischen und aus dem Handlungsdruck heraus verständlichen Position eine erweiterte (pflege-)ethische Reflexion dazuzustellen und in ein Diskussionspapier ${ }^{12}$ zu fassen. Dadurch sollten für die eine oder andere Entscheidung oder Konfliktlösung eine angemessene Begründung für die Kommunikation mit Bewohner*innen, de-

11 Die Stellungnahme vom 09.06.20 ist noch nicht online, aber über die Fachstelle SPES erhältlich; Kontaktdaten: https://www.im-muenchen.de/index.php?id=587.

12 Das schon erwähnte Papier konnte am 12.05.2020, dem Welttag der Pflege, veröffentlicht werden; an der Erstellung dieses Papiers hatten mitgewirkt: Heidi Albisser Schleger (Basel), Constanze Giese (München), Stefan Dinges (Wien), Georg Marckmann (München), Ingo Nordmann (Göttingen), Wolfgang Pasch (Düsseldorf), Marianne Rabe (Berlin), Annette Riedel (Esslingen), Nina Streeck (Zürich), Sabine Wöhlke (Göttingen): https://www.aem-online.de/fileadmin/user_upload/2 020_05_12_Pflegeethische_Reflexion_Papier.pdf [21.06.2020]. 
ren Angehörigen und den Mitarbeiter*innen gefunden werden. Im Idealfall, so die Überlegung, ließen sich noch Handlungsalternativen finden oder, so die Hoffnung, könne es zu einer geteilten Verantwortung zwischen Gesundheitspolitik und den Pflegeeinrichtungen kommen, die die Mitarbeiter*innen in ihrem moralischen Stress entlasten und unterstützen.

Das Diskussionspapier hat sich zur Aufgabe gemacht, auf die aktuell beobachtbaren Phänomene, Herausforderungen und deren Ursachen aufmerksam zu machen; insbesondere im ambulanten Bereich sowie in der stationären Langzeitpflege in Bezug auf die Beziehungsgestaltung, Kommunikation, Begleitung und die Wahrung von Rechten und Sicherheit der Bewohner"innen. In Spannung dazu wurden die aktuellen Arbeitsbedingungen in der Pflege sowie ethische Fragen gestellt, die sich für Pflegende in Zeiten der Covid-19-Pandemie ergeben (z. B. Pflege ohne ausreichende Schutzausrüstung vs. notwendige Berührungen und Zuwendung); in den Blick kamen auch Rahmenbedingungen für Auszubildende in den pflegenden Berufen und deren Einsatz in der Pflege vor Ort. Dazu gesellte sich auch der große Daten- und Forschungsbedarf aus der Sicht der Pflegewissenschaften, Public Health und Versorgungsforschung - zu wenige aktuelle Studien waren aus dieser Perspektive verfügbar. Die Beschränkungen in der Krise betreffen erneut jene Einrichtungen und Dienste, die bislang schon mit knapper Personaldecke und Ressourcenknappheit leben mussten - und das zeigt sich gerade durch fehlende Schutzausrüstungen sowie den Wegfall von unterstützenden Rollen und Angeboten wie Seelsorge, Physiotherapie, Ergotherapie etc.

Dem Diskussionspapier geht es darum, die verantwortlichen Träger, aber auch die Verbände und die Politik in den Blick zu nehmen und einen organisationsethischen Rahmen zur Bearbeitung der aktuellen Herausforderungen mitzuentwickeln. Den Hintergrund für die Positionen des Diskussionspapiers bildet eine solide pflegeethische Reflexion. Eine aktuell verantwortliche Pflegeethik darf sich hier nicht mit der Reflexion der eigenen Rolle und Profession begnügen; vielmehr geht es um das Ausgestalten einer interprofessionellen Ethik der Gesundheitsberufe bzw. des Gesundheitssystems. 


\section{Widersprüche und Spannungsfelder in der Corona-Krise}

Die Ethikberatung ist angetreten, Wert- und Zielkonflikte in Behandlungsund Versorgungsentscheidungen sichtbar und besprechbar zu machen. Im Sinne einer evaluativen Ethik gilt es, dabei konkurrierende Werte zu benennen, um dann in einer Güter- und Zielabwägung die Entscheidungen zu reflektieren. Gerade weil angesichts der drängenden Frage nach guter Versorgung und guten Entscheidungen auf der Mikroebene viele moralische Konflikte sichtbar werden, ist es ratsam, Perspektive und Ebene zu wechseln und auf einige Spannungsfelder und Widersprüche auf der Mesoebene der Gesundheitseinrichtungen im Lichte der gesellschaftlichen Gegenwartskultur (Makroebene) hinzuweisen. Es sind übrigens die gleichen Spannungsfelder und Widersprüchlichkeiten, die auch in Frankreich und Italien vorfindbar sind (z. B. die Privatisierungen im Gesundheitssektor und die gewinnorientierte Ausrichtung der Alten- und Pflegeheime, insbesondere in Norditalien). Das Ausbleiben der Überlastung in den Spitälern ist gegebenenfalls ein Unterschied zu den deutschsprachigen Gesundheitseinrichtungen; was nicht bedeutet, dass die deutschsprachigen Länder und Gesundheitseinrichtungen hier unverwundbar wären - das beweisen die vorgestellten Zahlen und Berichte aus den Pflegeeinrichtungen.

\subsection{Spannungsfelder und Aufgaben im Kontext des Gesundheitssystems}

Es braucht eine Organisation von Verantwortung zwischen den Trägern der stationären Altenpflege, den Berufsverbänden und der (kommunalen) Politik.

- Im Bereich der Krankenhäuser kam es in der Pandemie zu kommunalen bzw. regionalen Kooperationen bzw. der Koordination vorhandener Ressourcen ${ }^{13}$ : Unter der Wahrung notwendiger Schutzmaßnahmen und mit Rücksicht auf die Wünsche der Bewohner*innen ist auch im Bereich der stationären Altenpflege verstärkt über haus- und trägerübergreifende Kooperation und Koordination nachzudenken - gerne auch mit kommunalpolitischer Unterstützung bzw. Beauftragung. Aus

13 Auf der Homepage der DIVI findet sich ein Intensivregister aller freien Intensivbetten in Deutschland (https://www.intensivregister.de/\#/index [21.06.2020]). Wer die Mühen des Entlassungsmanagements in den Krankhäuser kennt, einen Pflege- oder Palliativplatz zu finden, fragt sich, warum es das noch nicht flächendeckend für den Pflegebedarf gibt. 
der Perspektive der Pflegethik werden in diesem Bereich im deutschsprachigen Raum entscheidungs- und handlungsfähige Krisenstäbe vermisst.

- In der öffentlichen Wahrnehmung sind die Pflegeheime das Problem, die Krankenhäuser jedoch die Lösungsanbieter?! - Hier tut ein Erkennen und Anerkennen Not, wie wenig der Altenpflegesektor (ambulant und stationär) bislang im Blick ist, wie ungleich die Aufmerksamkeit und die Anerkennung für Pflege- und Versorgungsleistungen sind. Die Krise sollte ein Anlass zu strukturellen Verbesserungen sein; dieser Diskurs muss jetzt auf der institutionellen und politischen Ebene geführt werden, nicht nur um aus der Corona-Krise herauszukommen, sondern um der bereits zuvor bestehenden kritischen Situation in der Langzeitpflege und in der ambulanten Pflege entgegenzuwirken.

Wie schon erwähnt, sollen die Maßnahmen in der Corona-Krise dem Schutz der alten und hochaltrigen Menschen dienen - in einem gesellschaftlichen Kontext, der strukturell altersdiskriminierend ist, ist das ein Widerspruch par excellence. Diese Diskriminierung ${ }^{14}$ trifft nicht nur strukturell die Einrichtungen der Altenpflege, sondern auch deren Mitarbeiter*innen und die zu Pflegenden.

- Aus Gründen der Gerechtigkeit, Fairness und Unparteilichkeit ist daran zu erinnern, dass die Mittel für Prävention, Pflege, Behandlung und Betreuung in einem solidarisch finanzierten Gesundheitssystem fair verteilt werden sollen. Wenn es um das Leben und die Gesundheit geht, gilt jede Gesundheitsinstitution gleich viel. Dies bedeutet u. a., dass die Langzeitpflege gegenüber der Akutpflege nicht nachrangig behandelt werden darf.

- Es ist anzuerkennen, dass die jetzige Krise frühere Fehlentscheidungen und Versäumnisse im Gesundheitswesen ans Tageslicht bringt und die Chance eröffnet, diese konstruktiv aufzugreifen und Veränderungen anzuregen. Berufs- und Fachverbände ebenso wie lokale und regionale Behörden und die entsprechenden politischen Organe sind gefordert, die Langzeitpflege gleichermaßen und angemessen in ihre Programme und Unterstützungsmaßnahmen zu integrieren.

- Akut braucht es (immer noch) Finanzmittel für Entlastungs- und für Schutzmaßnahmen. Die Frage der Finanzierung ließe sich bei diesem

14 Vgl. Rothermund/Mayer, Altersdiskriminierung; Schär-Bütikofer, Altersdiskriminierung in der Akutpflege. Sargent-Cox, Ageism: we are our own worst enemy; Dinges, „lebenssatt oder lebensmüde?“. 
Beispiel mit den verantwortlichen Stakeholdern aus Politik, Behörden, Krankenversicherungen oder Trägern in aller Kürze regeln. Das würde gut zum Stichwort „Koste es, was es wolle!“ passen: 50 Millionen Euro für ein Versorgungszentrum mit zusätzlichem Personal auf dem Wiener Messegelände waren kein Problem. ${ }^{15}$

- Mittelfristig sind alle Privatisierungskonzepte im Gesundheitswesen zu überdenken. Der Gesundheitssektor in Norditalien wurde massiven Einsparungen unterworfen durch Privatisierungen in Krankenhäusern und Altenheimen; in Frankreich wird das zentralistische Gesundheitssystem für die Eskalationen in der Krise verantwortlich gemacht. In Österreich sollte die regionale und kommunale Vernetzung weiter ausgebaut werden, gerne auch in Public-Private-Partnership.

\subsection{Zwischen Krankenhaus und Pflegeheim}

Im Hinblick auf eine mögliche Überlastung von Krankenhäusern wurden Patient*innen und Bewohner*innen verstärkt mit der Abfassung von Patientenverfügungen konfrontiert, um u. a. auf gebotene medizinischpflegerische Versorgung im Krankenhaus zu verzichten. So zentral Wunsch und Wille für jede Pflege und Versorgung sind, so darf jedenfalls kein Druck auf Willensäußerungen ausgeübt werden; gleichzeitig ist für sinnvoll erwünschte medizinisch-pflegerische Behandlungen zu sorgen wofür ja in den Krankenhäusern gesorgt worden war. Alter und Gebrechlichkeit allein dürfen weder Anlass noch Begründung von Rationierungsmaßnahmen sein; hier braucht es vorausschauende Wert- und Güterabwägungen, die sich mit und an den Bewohner*innen bzw. deren Lebenszielen orientieren.

Die freie Entscheidung des Einzelnen ist ein Grundrecht und eine ethische Maxime. Sie dürfte nur dann eingeschränkt werden, wenn mit alternativen Schritten zentrale Ziele bei der Vorbeugung und Behandlung von Covid-19 nicht erreicht werden können. Viele Bewohner*innen, die weder dement sind noch sich in gesetzlichen Vertretungen/Bevollmächtigungen befinden, konnten nicht in die Einschränkungen einwilligen bzw. wurde nicht um ihre Einwilligung gefragt. Damit stellt sich die Frage nach der Rechtsgrundlage für die Bewegungseinschränkungen bzw. Besuchs- und Begleitungsverbote von Angehörigen, Seelsorger*innen etc. für Bewohner*innen, die ganz offensichtlich weder ein Verdachtsfall noch infiziert

15 Vgl. https://wien.orf.at/stories/3052610/ [21.06.2020]. 
waren. Das stand und steht in Spannung zu jenen Bewohner*innen (und Angehörigen), die geschützt werden wollten - auch das eine Zerreißprobe für Mitarbeiter*innen und Einrichtungen. Trotzdem müssen viele der jetzt sichtbar werdenden Situationen mindestens als freiheitseinschränkende, wenn nicht freiheitsentziehende Maßnahmen bewertet werden und hätten dementsprechend nach den jeweils geltenden Gesetzeslagen gemeldet und überprüft werden müssen.

Im Vergleich zu den aufwändigen Kontrollen und Testungen in den Krankenhäusern wurde im niedergelassenen Bereich und in den Pflegeheimen zu wenig und zu zögerlich getestet; dies gilt für Bewohner*innen ebenso wie für die Mitarbeiter*innen. Die an sich gute Idee, z. B. in Wien über den Ärztefunkdienst die mobilen Tests zu organisieren und Infektionsketten zu verhindern, geriet bald in Überlastungen. Zwar wurde damit ein Ansturm auf die Spitäler verhindert; allerdings warteten viele tagelang auf den Test und dann wiederum auf das Ergebnis. In den Pflegeheimen wurde oft nur im Verdachtsfall oder nach einem Infektionsausbruch getestet.

Da es ja trotz Besuchs- und Ausgangsverboten immer wieder zu Infektionsherden in Pflegeheimen kam, waren es wohl die Mitarbeiter*innen, die das Virus in die Einrichtungen trugen. Mitarbeiter"innen verschwiegen ihre Symptome, hielten sie vielleicht nur für eine Grippe - und kamen zur Arbeit, nicht zuletzt auch, weil sie auf das Einkommen angewiesen waren oder um die Versorgungskontinuität bei Personalknappheit aufrechtzuerhalten. Hier hätte wohl ebenso konsequent getestet werden müssen, denn Mitarbeiter*innen-Sicherheit ist immer auch Patient"innen-/Bewohner*innen-Sicherheit.

Der Ressourcenmangel führte in manchen Pflegeeinrichtungen dazu, dass das Personal wegen des fehlenden Schutzmaterials mit Plastiksäcken und Skibrillen versucht hat, die Bewohner*innen und sich selbst vor einer Infektion zu schützen. Es stellt sich die Frage, ob nicht auch Mitarbeiter"innen das Recht haben, die Pflegearbeit ohne Schutz zu verweigern auch mit der Perspektive, dass eine Infektion von Pflegepersonen in Zeiten des Personalmangels eben auch die zukünftige Pflege und Versorgung in den Einrichtungen gefährdet.

\subsection{In den Pflegeheimen}

Die vorangegangenen Reflexionen machen deutlich, dass die zwiespältigen Erfahrungen und Konflikte in den Einrichtungen selbst durch Widersprüche und Wertkonflikte sowie Ungereimtheiten auf den übergeordneten 
Ebenen mitverursacht und mitverantwortet werden - und deswegen auf der Mikroebene der Alltagspflege nicht zufriedenstellend und nachhaltig gelöst werden können, ohne von den Mitarbeiter*innen herkulische Mehrleistungen einzufordern und Überlastungen vorzuprogrammieren.

Das Diskussionspapier der AEM würdigt explizit die geleistete Arbeit der Pflegenden in allen Gesundheitseinrichtungen und greift in den Vorbemerkungen einige Kernpositionen aus der Berufsethik der Pflege auf:

Gesunderhaltung und körperliche Unversehrtheit sind zentrale Pflegeziele $^{16}$ wie auch der Schutz und die Förderung der Autonomie des Menschen, der niemals nur Objekt der (Für-) Sorge sein darf, sondern immer Subjekt der ihn betreffenden Maßnahmen bleiben muss. ${ }^{17}$ Die Ermöglichung sozialer Teilhabe und von sozialen Nahbeziehungen ${ }^{18}$ wie auch die Bedeutung von Nähe und von Berührungen ${ }^{19}$ für den Menschen gehören zum Verantwortungs- und Kompetenzbereich der Pflege und sind zugleich zentrale Indikatoren von Pflegequalität. ${ }^{20}$

Die Erfahrungen und die Güter- und Wertkonflikte in der Pandemie stellten für viele Pflegende eine Bürde dar, weil der Schutz gegen Güter und Werte verstieß, die in einem ganzheitlich geprägten Pflegeprozess grundlegend sind: „Pflegende arbeiten ,am Leib ebenso wie mit dem Leib، und wissen deshalb um die scheinbar nur alltäglichen, aber zugleich doch lebenswichtigen Bedürfnisse des Menschen." 21 Somit standen etliche Handlungen der fachlichen und ethischen Expertise der Pflege als Profession und ihren Grundüberzeugungen entgegen: „Unsere physische Präsenz ist vielleicht das Kostbarste, was wir einander geben können. “22

16 Vgl. ICN-Ethikkodex für Pflegende 2012 (deutsche Fassung von 2014), Präambel, in: https://www.dbfk.de/media/docs/download/Allgemein/ICN-Ethikkodex-2012deutsch.pdf [21.06.2020].

17 Vgl. ICN-Ethikkodex, Element 1; explizit auch in: Charta zur Betreuung schwerstkranker und sterbender Menschen in Deutschland, Leitsatz 1, unter: https:/www. charta-zur-betreuung-sterbender.de/die-charta_leitsaetze.html [21.06.2020].

18 Vgl. ICN-Ethikkodex, Element 1; Pflege-Charta, Artikel 4, Artikel 6, Artikel 8, unter: https://www.wege-zur-pflege.de/pflege-charta/ [21.06.2020].

19 Gertzen, Streicheleinheiten im Altersheim.

20 https://www.aem-online.de/fileadmin/user_upload/2020_05_12_Pflegeethische_R eflexion_Papier.pdf [21.06.2020].

21 Großklaus-Seidel, Ethik im Pflegealltag, 96; sie zitiert Hartmut Remmers.

22 Guiver, Company of voices, 35. 
Das Diskussionspapier nimmt einige der zwiespältigen Erfahrungen im Pflegealltag auf - hier einige, die bislang noch unerwähnt blieben:

- Durch die Priorisierung des Infektionsschutzes wurden Alltagsprozesse und Tagesstrukturen verändert, es kam zu erzwungenen Umzügen, Mahlzeiten wurden nicht mehr gemeinsam eingenommen und Gruppenaktivitäten massiv eingeschränkt.

- Insbesondere bei Menschen mit Demenz verschlimmerte sich die Verwirrtheit und in der Folge kam es zu einer Verschlechterung des Allgemeinzustandes, aber auch zu Aggressionen.

- Freiheitseinschränkende Maßnahmen aus der Überforderung der Pflegenden heraus bergen auch die Gefahr einer Zunahme von Gewalt und Übergriffen.

- Es konnte eine Zunahme in der Verabreichung ruhigstellender Medikamente beobachtet werden, die nicht aus therapeutischen Gründen, sondern aus einer Überlastung heraus verabreicht wurden.

- Die latente Gefahr von Unter- bzw. Fehlversorgung wurde durch unzureichend eingearbeitetes Aushilfspersonal gesteigert.

\section{Die Forderungen und Empfehlungen des AEM-Diskussionspapiers}

Abschließend übernehme ich die Forderungen und Empfehlungen aus pflegeethischer Sicht und ihre Konsequenzen auf den unterschiedlichen Ebenen aus dem zitierten Dokument:

- „Menschenrechte und Grundrechte gelten für Pflegebedürftige wie für alle Menschen auch in Zeiten der Covid-19-Pandemie.

- Eine qualitativ hochwertige Patientenversorgung und Pflege hat stets Respekt vor der Würde einer jeden Person.

- Das gesellschaftliche Potenzial der Pflegeberufe liegt nicht in ihrer Aufopferungsbereitschaft, sondern in ihrer fachlichen Expertise, die den Carebedarf der Bürger*innen vertritt.

- Die gesellschaftliche und politische Anerkennung professioneller Pflege und der Pflegenden muss über die Covid-19-Pandemie und über das Internationale Jahr der Pflegenden hinauswirken. Nicht einmalige Bonuszahlungen, sondern langfristige finanzielle, soziale, gesellschaftliche und interprofessionelle Anerkennung sind gefordert.

- Die andauernde pflegerische Versorgungskrise, die durch die Covid-19Pandemie weiter eskaliert, führt eindrücklich vor Augen, dass der politische Verzicht auf eine Verkammerung der Pflege in fast allen Bundes- 
ländern keine ethisch neutrale politische Entscheidung war und korrigiert werden muss.

- Das Spannungsfeld zwischen Infektionsgefahr, leiblicher Nähe und Lebensqualität, in dem Pflegende sich bewegen, bedarf adäquater Strukturen der ethischen Reflexion.

- Wenn es um das Leben und die Gesundheit geht, gilt es, jedem Versorgungssektor des Gesundheitswesens in seiner Bedeutung gerecht zu werden und die Langzeitpflege nicht länger nachrangig zu behandeln. Dazu muss lokale und kommunale sektorenübergreifende Vernetzung beitragen.

- Nicht nur die Pflegenden mit Patientenkontakt, sondern auch die Institutionen (Krankenhäuser, Senioreneinrichtungen, ambulante Pflegedienste, private und staatliche Einrichtungen sowie die übergeordneten Behörden und politischen Organe) sind moralische Akteure, die ihre je eigene moralische Verantwortung wahrnehmen müssen. “23

Auf der Mikroebene können diese Forderungen umgesetzt werden durch:

- eine professionelle Verantwortungsübernahme der Pflegenden vor Ort und ihre vorausschauende Einbindung bei der Gestaltung aller Versorgungsprozesse;

- eine Sorge für Rechtssicherheit, nicht nur im Umgang mit Freiheitsbeschränkungen;

- eine Sorge für eine angemessene Ausstattung und Ausrüstung für die zentralen Pflegeprozesse.

Auf der Mesoebene der Gesundheitseinrichtungen verlangt dies u. a.:

- die Organisation eines Pandemiestabes;

- eine routinemäßige Überprüfung aller bestehenden Freiheitseinschränkungen und

- die Sicherstellung einer würdebewahrenden Begleitung und Versorgung am Lebensende.

Auf der gesellschaftlichen Ebene braucht dies endlich eine Beteiligung der Pflegeberufe und ihrer Standesvertretungen in allen Belangen der Gesundheitsversorgung, nicht nur weil sie immer in der Umsetzung unmittelbar beteiligt sind, sondern auch weil sie in ihrer Professionalisierung längst schon einen zentralen und eigenständigen Beitrag leisten, der unverzichtbar ist. Es wird deutlich, dass nicht nur die Risikogruppen unsere Sorge

23 https://www.aem-online.de/fileadmin/user_upload/2020_05_12_Pflegeethische_R eflexion_Papier.pdf [21.06.2020]. 
brauchen, wir müssen uns auch angemessen um die Gesundheitsberufe sorgen, damit sie ihr professionelles Können zu unser aller Wohl umsetzen können. Letztendlich profitiert jede einzelne Bürgerin, jeder einzelne Bürger von einem solidarischen, ethisch aufmerksamen und auf den Einzelfall fokussierten Gesundheitssystem - in gesellschaftlicher Mitverantwortung.

\section{Literatur}

Dinges, Stefan: Ethikberatung als Prävention von moralischem Stress im Bereich von Pflege- und Gesundheitsberufen, in: Eisele, Colombine (Hg.): Moralischer Stress in der Pflege. Auseinandersetzung mit ethischen Dilemmasituationen, Wien: Facultas 2017, 76-90.

Dinges, Stefan: „lebenssatt oder lebensmüde?“ Verstehen, analysieren und bearbeiten der Sterbewünsche von Bewohner*innen in der stationären Altenhilfe, Linz 2019 (= unveröffentlichte Masterthesis zur Erlangung eines Professional Master in Medical Ethics (PM.ME) an der Johannes Kepler Universität).

Dinges, Stefan: Eine Sorge für das Ganze (Oıkovopía): Welchen Beitrag Pflegende für eine organisationsethische Ausrichtung von Gesundheitseinrichtungen leisten - trotz moralischem Stress, Rollenkonflikten und ungeklärter Verantwortung, in: Stronegger Willibald J./Attems, Kristin (Hg.): Das Lebensende zwischen Ökonomie und Ethik. 2. Goldegger Dialogforum Mensch und Endlichkeit, Baden-Baden: Nomos 2019 (= Bioethik in Wissenschaft und Gesellschaft 7), 147-166.

Gertzen, Ann-Christin: Streicheleinheiten im Altersheim. Die Kunst der Berührung, Süddeutsche Zeitung (17.05.2010), in: https://www.sueddeutsche.de/leben /streicheleinheiten-im-altersheim-die-kunst-der-beruehrung-1.359960 [21.06.2020].

Großklaus-Seidel, Marion: Ethik im Pflegealltag, Stuttgart: Kohlhammer 2002.

Guiver George: Company of voices. Daily prayer and the people of God, Norwich: Canterbury 2000.

Rothermund, Klaus/Mayer Anne-Kathrin: Altersdiskriminierung. Erscheinungsformen, Erklärungen und Interventionsansätze, Stuttgart: Kohlhammer 2009.

Schär-Bütikofer, Ursula: Altersdiskriminierung in der Akutpflege. Auf den Spuren eines Phänomens, München: GRIN 2010.

Sargent-Cox, Kerry: Ageism: we are our own worst enemy, in: International Psychogeriatrics 29/1 (2017) 1-8. 